\title{
ON THE OPTIMAL CONTINUED FRACTION EXPANSION OF A QUADRATIC SURD
}

\author{
KEITH R. MATTHEWS \\ (Received 3 December 2010; accepted 1 February 2012; first published online 7 February 2013) \\ Communicated by J. O. Shallit \\ Dedicated to the memory of Alf van der Poorten
}

\begin{abstract}
We describe the period structure of the optimal continued fraction expansion of a quadratic surd, in terms of the period of its nearest square continued fraction expansion. The analysis results in a faster algorithm for determining the optimal continued fraction expansion of a quadratic surd.
\end{abstract}

2010 Mathematics subject classification: primary 11A55; secondary 11Y65.

Keywords and phrases: optimal continued fraction, nearest square continued fraction, quadratic surd, period length.

\section{Introduction}

In 1960, Selenius [7] introduced a new semi-regular continued fraction (SK) expansion of a real number, arising from its regular continued fraction (RCF) expansion. Like the RCF, Selenius' continued fraction is periodic for quadratic surds. Selenius discussed the SK expansion of $\sqrt{D}$ in detail, describing its period in terms of that of the nearest square continued fraction (NSCF) of Ayyangar [1]. Bosma and Kraaikamp [3] studied a more accessible version of Selenius' continued fraction, namely the optimal continued fraction (OCF) of Bosma [2] and also discussed periodicity for a quadratic surd. The OCF algorithm is defined in Section 2, independently of the RCF algorithm. However, in Section 3 we present the OCF in terms of the RCF, in such as way as to emphasize similarities with the NSCF algorithm. A connection between the OCF and NSCF is made in Section 6 using work of Selenius, and in Section 8 we give an explicit description of an OCF period of a quadratic surd in terms of its NSCF period. In Section 9 our analysis results in a faster algorithm for determining the OCF expansion of a quadratic surd, which starts with the Bosma algorithm and soon switches to the NSCF algorithm, with perturbations in exceptional cases. This second stage avoids

(C) 2013 Australian Mathematical Publishing Association Inc. 1446-7887/2013 \$16.00 
the time-consuming calculation of a second integer part in the Bosma algorithm at step (2.3) below.

\section{The optimal continued fraction}

Definition 2.1 [2]. The OCF expansion of an irrational number $\xi_{0}$ is defined recursively as follows: first, $r_{-1}=1, r_{-2}=0, s_{-1}=0, s_{-2}=1, \epsilon_{0}=1$. Then for $k \geq 0$, with $\tilde{\xi}_{0}=\xi_{0}$,

$$
\begin{aligned}
b_{k} & =\left\lfloor\tilde{\xi}_{k}\right\rfloor \\
\frac{u_{k}}{v_{k}} & =\frac{b_{k} r_{k-1}+\epsilon_{k} r_{k-2}}{b_{k} s_{k-1}+\epsilon_{k} s_{k-2}} \\
\beta_{k} & =v_{k} /\left(2 v_{k}+s_{k-1}\right) \\
\tilde{a}_{k} & =\left\lfloor\tilde{\xi}_{k}+\beta_{k}\right\rfloor \\
\frac{r_{k}}{s_{k}} & =\frac{\tilde{a}_{k} r_{k-1}+\epsilon_{k} r_{k-2}}{\tilde{a}_{k} s_{k-1}+\epsilon_{k} s_{k-2}} \\
t_{k} & =\tilde{\xi}_{k}-\tilde{a}_{k} \\
\epsilon_{k+1} & =\operatorname{sign}\left(t_{k}\right) \\
\tilde{\xi}_{k+1} & =\epsilon_{k+1} / t_{k},
\end{aligned}
$$

where $\lfloor\cdot\rfloor$ denotes the integer part.

For $k \geq 0$, the OCF complete quotients satisfy $\tilde{\xi}_{k}=\tilde{a}_{k}+\epsilon_{k+1} / \tilde{\xi}_{k+1}$, with convergents $r_{k} / s_{k}$. We give some properties of the OCF.

Lemma 2.2. With $b_{k}$ and $\tilde{a}_{k}$ defined as in (2.1) and (2.3),

$$
\begin{gathered}
\tilde{a}_{k}= \begin{cases}b_{k} & \text { if } \epsilon_{k+1}=1, \\
b_{k}+1 & \text { if } \epsilon_{k+1}=-1 .\end{cases} \\
\frac{r_{k}}{s_{k}}= \begin{cases}\frac{u_{k}}{v_{k}} & \text { if } \epsilon_{k+1}=1, \\
\frac{u_{k}+r_{k-1}}{v_{k}+s_{k-1}} & \text { if } \epsilon_{k+1}=-1 .\end{cases}
\end{gathered}
$$

If $A / B$ is a rational number with $B>0$ and $H(A / B)=B\left|B \xi_{0}-A\right|$, then

$$
\epsilon_{k+1}= \begin{cases}1 & \text { if } H\left(\frac{u_{k}}{v_{k}}\right)<H\left(\frac{u_{k}+r_{k-1}}{v_{k}+s_{k-1}}\right), \\ -1 & \text { if } H\left(\frac{u_{k}}{v_{k}}\right)>H\left(\frac{u_{k}+r_{k-1}}{v_{k}+s_{k-1}}\right) .\end{cases}
$$


Proof. Equation (2.8) is stated in [2, Lemma (4.6)]. Equation (2.9) follows from (2.4), (2.8) and (2.2). Equation (2.10) follows from [2, Lemma (4.3)], which states that

together with (2.9).

$$
\frac{r_{k}}{s_{k}}=\frac{u_{k}}{v_{k}} \Longleftrightarrow H\left(\frac{u_{k}}{v_{k}}\right)<H\left(\frac{u_{k}+r_{k-1}}{v_{k}+s_{k-1}}\right),
$$

\section{The OCF algorithm via the RCF algorithm}

In this section we show how the OCF expansion of an irrational number $\xi_{0}$ may be derived from the RCF expansion $\xi_{0}=a_{0}+1|/| a_{1}+\cdots$, using the function $H$.

We recall that the $n$th RCF complete quotient $\xi_{n}$ is defined for $n \geq 0$ by

$$
a_{n}=\left\lfloor\xi_{n}\right\rfloor \text { and } \xi_{n}=a_{n}+1 / \xi_{n+1} .
$$

The $n$th RCF convergent $A_{n} / B_{n}$ is defined for $n \geq-2$ by $A_{-2}=0, A_{-1}=1, B_{-2}=1$, $B_{-1}=0$ and, for $k \geq-1$,

$$
\begin{aligned}
& A_{k+1}=a_{k+1} A_{k}+A_{k-1}, \\
& B_{k+1}=a_{k+1} B_{k}+B_{k-1} .
\end{aligned}
$$

LeMma 3.1. Let $h(k)$ be defined recursively by $h(0)=0$ and

$$
h(k+1)= \begin{cases}h(k)+1 & \text { if } \epsilon_{k+1}=1, \\ h(k)+2 & \text { if } \epsilon_{k+1}=-1 .\end{cases}
$$

Then

$$
b_{k}= \begin{cases}a_{h(k)} & \text { if } \epsilon_{k}=1, \\ a_{h(k)}+1 & \text { if } \epsilon_{k}=-1,\end{cases}
$$

and

$$
\tilde{\xi}_{k}= \begin{cases}\xi_{h(k)} & \text { if } \epsilon_{k}=1, \\ \xi_{h(k)}+1 & \text { if } \epsilon_{k}=-1 .\end{cases}
$$

Proof. We prove (3.2) and (3.3) by induction on $k \geq 0$. These are clearly true when $k=0$, as $h(0)=0$ and $\epsilon_{0}=1$, so $\tilde{\xi}_{0}=\xi_{0}=\xi_{h(0)}$ and $b_{0}=\left\lfloor\tilde{\xi}_{0}\right\rfloor=\left\lfloor\xi_{0}\right\rfloor=a_{0}=a_{h(0)}$. We now assume that (3.2) and (3.3) hold. Now by (2.7), $\tilde{\xi}_{k+1}=\epsilon_{k+1} /\left(\tilde{\xi}_{k}-\tilde{a}_{k}\right)$.

Case 1. Assume that $\epsilon_{k+1}=1$. Then using (2.8) and (3.2),

$$
\begin{aligned}
& \tilde{\xi}_{k+1}=\frac{1}{\tilde{\xi}_{k}-\tilde{a}_{k}}=\frac{1}{\tilde{\xi}_{k}-b_{k}}= \begin{cases}\frac{1}{\xi_{h(k)}-a_{h(k)}} & \text { if } \epsilon_{k}=1, \\
\frac{1}{\left(\xi_{h(k)}+1\right)-\left(a_{h(k)}+1\right)} & \text { if } \epsilon_{k}=-1,\end{cases} \\
& =\frac{1}{\xi_{h(k)}-a_{h(k)}}=\xi_{h(k)+1}=\xi_{h(k+1)},
\end{aligned}
$$

by (3.1). 
Case 2. Assume that $\epsilon_{k+1}=-1$. Then, again using (2.8) and (3.2),

$$
\begin{aligned}
\tilde{\xi}_{k+1}=\frac{-1}{\tilde{\xi}_{k}-\tilde{a}_{k}}=\frac{-1}{\tilde{\xi}_{k}-\left(b_{k}+1\right)} & = \begin{cases}\frac{-1}{\xi_{h(k)}-\left(a_{h(k)}+1\right)} & \text { if } \epsilon_{k}=1, \\
\frac{-1}{\left(\xi_{h(k)}+1\right)-\left(a_{h(k)}+2\right)} & \text { if } \epsilon_{k}=-1,\end{cases} \\
& =\frac{-1}{\xi_{h(k)}-a_{h(k)}-1}=\frac{-1}{\frac{1}{\xi_{h(k)+1}}-1} .
\end{aligned}
$$

But by Equation (4.17) of [2, Proposition 4.14], namely

$$
a_{h(k)+1}=1 \text { if } \epsilon_{k+1}=-1,
$$

we have $\xi_{h(k)+1}=1+1 / \xi_{h(k)+2}$. Hence by (3.4),

$$
\tilde{\xi}_{k+1}=\frac{-1}{\frac{\xi_{h(k)+2}}{\xi_{h(k)+2}+1}-1}=\xi_{h(k)+2}+1=\xi_{h(k+1)}+1,
$$

by (3.1). Thus we have shown that (3.3) holds when $k$ is replaced by $k+1$.

Also, by (2.1),

$$
\begin{aligned}
b_{k+1}=\left\lfloor\tilde{\xi}_{k+1}\right\rfloor & = \begin{cases}\left\lfloor\xi_{h(k+1)}\right\rfloor & \text { if } \epsilon_{k+1}=1, \\
\left\lfloor\xi_{h(k+1)}\right\rfloor+1 & \text { if } \epsilon_{k+1}=-1,\end{cases} \\
& = \begin{cases}a_{h(k+1)} & \text { if } \epsilon_{k+1}=1, \\
a_{h(k+1)}+1 & \text { if } \epsilon_{k+1}=-1 .\end{cases}
\end{aligned}
$$

Hence (3.2) holds when $k$ is replaced by $k+1$.

We now see from Lemmas 2.2 and 3.1 how the OCF can be derived from the RCF, in terms of the function $H$. With $\epsilon_{0}=1, h(0)=0, r_{-2}=0, r_{-1}=1, s_{-2}=1, s_{-1}=0$ and assuming that $\epsilon_{k}, h(k), r_{k-1}, s_{k-1}, r_{k-2}$ and $s_{k-2}$ are defined, we define $\epsilon_{k+1}, h(k+1)$ and $r_{k} / s_{k}$ as follows. Let

$$
\begin{gathered}
b_{k}= \begin{cases}a_{h(k)} & \text { if } \epsilon_{k}=1, \\
a_{h(k)}+1 & \text { if } \epsilon_{k}=-1,\end{cases} \\
\frac{u_{k}}{v_{k}}=\frac{b_{k} r_{k-1}+\epsilon_{k} r_{k-2}}{b_{k} s_{k-1}+\epsilon_{k} s_{k-2}}, \\
\epsilon_{k+1}= \begin{cases}1 & \text { if } H\left(\frac{u_{k}}{v_{k}}\right)<H\left(\frac{u_{k}+r_{k-1}}{v_{k}+s_{k-1}}\right), \\
-1 & \text { if } H\left(\frac{u_{k}}{v_{k}}\right)>H\left(\frac{u_{k}+r_{k-1}}{v_{k}+s_{k-1}}\right),\end{cases}
\end{gathered}
$$




$$
\begin{gathered}
\frac{r_{k}}{s_{k}}= \begin{cases}\frac{u_{k}}{v_{k}} & \text { if } \epsilon_{k+1}=1, \\
\frac{u_{k}+r_{k-1}}{v_{k}+s_{k-1}} & \text { if } \epsilon_{k+1}=-1,\end{cases} \\
h(k+1)= \begin{cases}h(k)+1 & \text { if } \epsilon_{k+1}=1, \\
h(k)+2 & \text { if } \epsilon_{k+1}=-1 .\end{cases}
\end{gathered}
$$

Then $\tilde{a}_{k}$ and $\tilde{\xi}_{k}$ can be defined by (2.8) and (3.3).

Remark 3.2. Bosma [2] used the notation $n(k)$, rather than $h(k)$. Let $n(k)=h(k+1)-1$ for $k \geq-1$ and define $n(-2)=-2$. Then, for $k \geq-1$, it follows from (3.1) that

$$
n(k)= \begin{cases}n(k-1)+1 & \text { if } \epsilon_{k+1}=1, \\ n(k-1)+2 & \text { if } \epsilon_{k+1}=-1 .\end{cases}
$$

Lemma 3.3. For $k \geq-2$,

$$
r_{k}=A_{n(k)}, \quad s_{k}=B_{n(k)} .
$$

Proof. This was proved for $k \geq 1$ in [2, Proposition (4.14)] and is trivially true for $k=-1$ and -2 . It is also true for $k=0$. For $s_{0}=1$, (i) $\epsilon_{1}=1$ implies that $n(0)=0$ and $B_{n(0)}=B_{0}=1$; whereas (ii) if $\epsilon_{1}=-1$, we have $n(0)=1$ and $B_{n(0)}=B_{1}=a_{1}$. Then (2.10) with $k=0$ gives $H\left(b_{0} / 1\right)>H\left(\left(b_{0}+1\right) / 1\right)$, which implies that $\xi_{0}>a_{0}+$ $1 / 2$, from which it follows that $a_{1}=\left\lfloor 1 /\left(\xi_{0}-a_{0}\right)\right\rfloor=1$ and again $B_{1}=1$.

Similarly,

$$
r_{0}=\tilde{a}_{0}=\left\lfloor\xi_{0}+1 / 2\right\rfloor= \begin{cases}a_{0}=A_{0} & \text { if } \epsilon_{1}=1, \\ a_{0}+1=A_{1} & \text { if } \epsilon_{1}=-1 .\end{cases}
$$

Hence $r_{0}=A_{n(0)}$.

We note in passing that $s_{k}>s_{k-1}$ for $k \geq 1$ (see [2, Lemma (4.5)]) and that $s_{0}=1$. Also, in [2, Corollary (4.27)], Bosma showed that $\tilde{a}_{k} \geq 2$ for $k \geq 1$ and that $\tilde{\xi}_{m}>$ $(1+\sqrt{5}) / 2$ if $m \geq 1$, by [2, Proposition (4.21)].

The OCF expansion of a quadratic surd is eventually periodic, that is to say, $\epsilon_{i+1}=\epsilon_{i+p+1}$ and $\tilde{a}_{i}=\tilde{a}_{i+p}$ for all $i \geq i_{0}$ (see [3, Theorem (6.1)]). The least such $p$ is called the period length.

From now on, $\xi_{0}=\left(P_{0}+\sqrt{D}\right) / Q_{0}$ is assumed to be a quadratic irrationality in standard form, that is, $P_{0}, Q_{0}$ and $\left(D-P_{0}^{2}\right) / Q_{0}$ are integers whose greatest common divisor is equal to 1 . Then if the RCF complete quotient $\xi_{n}=\left(P_{n}+\sqrt{D}\right) / Q_{n}$ is in standard form, we have the positive and negative representations

$$
\xi_{n}=\left(P_{n}+\sqrt{D}\right) / Q_{n}=a_{n}+\frac{Q_{n+1}}{P_{n+1}+\sqrt{D}}=a_{n}+1-\frac{Q_{n+1}^{\prime \prime}}{P_{n+1}^{\prime \prime}+\sqrt{D}},
$$

where $\left(P_{n+1}+\sqrt{D}\right) / Q_{n+1}>1$ and $\left(P_{n+1}^{\prime \prime}+\sqrt{D}\right) / Q_{n+1}^{\prime \prime}>1$ are also in standard form. 
Lemma 3.4 (Selenius). Let $\xi_{0}$ be a quadratic irrational. Consider the positivenegative representation of $R C F$ complete quotient $\xi_{v-1}$ :

$$
\xi_{v-1}=\left(P_{v-1}+\sqrt{D}\right) / Q_{v-1}=a_{v-1}+\frac{Q_{v}}{P_{v}+\sqrt{D}}=a_{v-1}+1-\frac{Q_{v}^{\prime \prime}}{P_{v}^{\prime \prime}+\sqrt{D}} .
$$

(i) If $a_{v}=1$ and $v \geq 1$, then:
(a) $Q_{v}^{\prime \prime}=Q_{v+1}$;
(b) $P_{v}^{\prime \prime}=P_{v+1}+Q_{v+1}$.

(ii) If $\xi_{v-1}$ is $R C F$ reduced, then $Q_{v}^{\prime \prime} \leq Q_{v}$ implies that $a_{v}=1$.

Proof. See [7, Satz 37], where the results are proved for $\xi_{0}=\sqrt{D}$.

By (3.1) and (3.3), we can now view the RCF to OCF transformation as a sequence of traversals of the sequence of RCF positive and negative representations of $\xi_{h(k)}$ :

$$
\xi_{h(k)}=a_{h(k)}+\frac{Q_{h(k)+1}}{P_{h(k)+1}+\sqrt{D}}=a_{h(k)}+1-\frac{Q_{h(k)+1}^{\prime \prime}}{P_{h(k)+1}^{\prime \prime}+\sqrt{D}},
$$

visiting the $\xi_{h(k)}$, where $k \geq 0$, in jumps of 1 or 2 and choosing

$$
\tilde{\xi}_{k+1}= \begin{cases}\left(P_{h(k)+1}+\sqrt{D}\right) / Q_{h(k)+1} & \text { if } \epsilon_{k+1}=1, \\ \left(P_{h(k)+1}^{\prime \prime}+\sqrt{D}\right) / Q_{h(k)+1}^{\prime \prime} & \text { if } \epsilon_{k+1}=-1 .\end{cases}
$$

For if $\epsilon_{k+1}=1$, then

$$
\tilde{\xi}_{k+1}=\left(P_{h(k+1)}+\sqrt{D}\right) / Q_{h(k+1)}=\left(P_{h(k)+1}+\sqrt{D}\right) / Q_{h(k)+1} .
$$

Also if $\epsilon_{k+1}=-1$ then $h(k+1)=h(k)+2$. Moreover, by (3.5), $\epsilon_{k+1}=-1$ implies that $a_{h(k)+1}=1$, so by Lemma 3.4(i),

$$
P_{h(k)+1}^{\prime \prime}=P_{h(k)+2}+Q_{h(k)+2} \quad \text { and } \quad Q_{h(k)+1}^{\prime \prime}=Q_{h(k)+2},
$$

so

$$
\tilde{\xi}_{k+1}=\left(P_{h(k+1)}+\sqrt{D}\right) / Q_{h(k+1)}=\left(P_{h(k)+2}+\sqrt{D}\right) / Q_{h(k)+2}+1=\left(P_{h(k)+1}^{\prime \prime}+\sqrt{D}\right) / Q_{h(k)+1}^{\prime \prime} .
$$

Lemma 3.5. If $a_{n}>1$ for some $n \geq 1$, then $n=h(k)$ for some $k \geq 1$.

Proof. Let $a_{n}>1$ and $h(k) \leq n<h(k+1)$. If $h(k)<n$, then $h(k+1)=h(k)+2$ and $\epsilon_{k+1}=-1$; also $n=h(k)+1$. Hence, by (3.5), we get the contradiction $a_{n}=$ $a_{h(k)+1}=1$.

ExAmple 3.6. The initial OCF expansion of $\xi_{0}=(36+\sqrt{405}) / 81$ in given in Table 2 . This is derived from the positive-negative representations of the RCF expansion in Table 1, with line traversals indicated by $0 \rightarrow 2 \rightarrow 3 \rightarrow 5 \rightarrow 6 \rightarrow 7 \rightarrow 9 \rightarrow$ 11 , corresponding to $h(1)=2, h(2)=3, h(3)=5, h(4)=6, h(5)=7, h(6)=9$ and $h(7)=11$. 
TABLE 1. Positive-negative representations for $\xi_{0}=(36+\sqrt{405}) / 81$

\begin{tabular}{rccc}
\hline$i$ & $\xi_{i}$ & $a_{i}+Q_{i+1} /\left(P_{i+1}+\sqrt{D}\right)$ & $a_{i}+1-Q_{i+1}^{\prime \prime} /\left(P_{i+1}^{\prime \prime}+\sqrt{D}\right)$ \\
\hline 0 & $(36+\sqrt{405}) / 81$ & $0-11 /(-36+\sqrt{405})$ & $1-20 /(45+\sqrt{405})$ \\
1 & $(-36+\sqrt{405}) /(-11)$ & $1+20 /(25+\sqrt{405})$ & $2-19 /(14+\sqrt{405})$ \\
2 & $(25+\sqrt{405}) / 20$ & $2+9 /(15+\sqrt{405})$ & $3-41 /(35+\sqrt{405})$ \\
3 & $(15+\sqrt{405}) / 9$ & $3+29 /(12+\sqrt{405})$ & $4-4 /(21+\sqrt{405})$ \\
4 & $(12+\sqrt{405}) / 29$ & $1+4 /(17+\sqrt{405})$ & $2-59 /(46+\sqrt{405})$ \\
5 & $(17+\sqrt{405}) / 4$ & $9+11 /(19+\sqrt{405})$ & $10-31 /(23+\sqrt{405})$ \\
6 & $(19+\sqrt{405}) / 11$ & $3+19 /(14+\sqrt{405})$ & $4-20 /(25+\sqrt{405})$ \\
7 & $(14+\sqrt{405}) / 19$ & $1+20 /(5+\sqrt{405})$ & $2-9 /(24+\sqrt{405})$ \\
8 & $(5+\sqrt{405}) / 20$ & $1+9 /(15+\sqrt{405})$ & $2-41 /(35+\sqrt{405})$ \\
9 & $(15+\sqrt{405}) / 9$ & $3+29 /(12+\sqrt{405})$ & $4-4 /(21+\sqrt{405})$ \\
10 & $(12+\sqrt{405}) / 29$ & $1+4 /(17+\sqrt{405})$ & $2-59 /(46+\sqrt{405})$ \\
11 & $(17+\sqrt{405}) / 4$ & $9+11 /(19+\sqrt{405})$ & $10-31 /(23+\sqrt{405})$ \\
\hline
\end{tabular}

TABLE 2. Initial segment of the OCF expansion of $(36+\sqrt{405}) / 81$

\begin{tabular}{ccrc}
\hline$i$ & $\tilde{\xi}_{i}$ & $\epsilon_{i}$ & $\tilde{a}_{i}$ \\
\hline 0 & $(36+\sqrt{405}) / 81$ & 1 & 1 \\
1 & $(45+\sqrt{405}) / 20$ & -1 & 3 \\
2 & $(15+\sqrt{405}) / 9$ & 1 & 4 \\
3 & $(21+\sqrt{405}) / 4$ & -1 & 10 \\
4 & $(19+\sqrt{405}) / 11$ & 1 & 3 \\
5 & $(14+\sqrt{405}) / 19$ & 1 & 2 \\
6 & $(24+\sqrt{405}) / 9$ & -1 & 5 \\
7 & $(21+\sqrt{405}) / 4$ & -1 & 10 \\
\hline & & &
\end{tabular}

\section{Selenius' algorithm}

We need to show that the OCF algorithm is identical to Selenius' algorithm, as originally presented by Selenius [7, Section 27, V 4]. We start by identifying the quotients $u_{k} / v_{k}$ and $\left(u_{k}+r_{k-1}\right) /\left(v_{k}+s_{k-1}\right)$ that occur in Equation (2.9) of the OCF algorithm.

Lemma 4.1. Let $h(k)$ be defined as in (3.1). Let $h(k)=r$ and $\Theta_{r}=H\left(A_{r} / B_{r}\right)$. Then

$$
\frac{u_{k}}{v_{k}}=\frac{A_{r}}{B_{r}}, \quad \frac{u_{k}+r_{k-1}}{v_{k}+s_{k-1}}=\frac{A_{r}+A_{r-1}}{B_{r}+B_{r-1}} .
$$


Proof. Observe that

$$
\begin{aligned}
u_{k} & =b_{k} r_{k-1}+\epsilon_{k} r_{k-2} \\
& =b_{k} A_{n(k-1)}+\epsilon_{k} A_{n(k-2)} \\
& =b_{k} A_{r-1}+\epsilon_{k} A_{n(k-2)} .
\end{aligned}
$$

Case 1. Assume that $\epsilon_{k}=1$. Then from (3.2), $b_{k}=a_{h(k)}=a_{r}$ and

$$
h(k-1)=h(k)-1=r-1, \quad n(k-2)=r-2
$$

and (4.2) becomes $u_{k}=a_{r} A_{r-1}+A_{r-2}=A_{r}$.

Case 2. Assume that $\epsilon_{k}=-1$. Then $b_{k}=a_{r}+1$. Also by (3.5), $a_{h(k-1)+1}=1$, where $h(k-1)=h(k)-2=r-2$. Hence $a_{r-1}=1$ and $A_{r-1}=a_{r-1} A_{r-2}+A_{r-3}=A_{r-2}+A_{r-3}$.

Then (4.2) becomes $u_{k}=\left(a_{r}+1\right) A_{r-1}-A_{r-3}=a_{r} A_{r-1}+A_{r-2}=A_{r}$. Also in both cases

$$
u_{k}+r_{k-1}=A_{r}+A_{n(k-1)}=A_{r}+A_{h(k)-1}=A_{r}+A_{r-1},
$$

as required.

Corollary 4.2. Let $h(k)=r$ and $\Theta_{r}=H\left(A_{r} / B_{r}\right)$. Then:

(a) $\epsilon_{k+1}=1$ and $a_{r+1}=1$ implies that $\Theta_{r}<\Theta_{r+1}$;

(b) $\epsilon_{k+1}=-1$ implies that $\Theta_{r}>\Theta_{r+1}$.

Proof. This follows from (4.1) and (2.9), as $\left(u_{k}+r_{k-1}\right) /\left(v_{k}+s_{k-1}\right)=A_{r+1} / B_{r+1}$, if $a_{r+1}=1$. This is indeed the case if $\epsilon_{k+1}=-1$, as by (3.5), $a_{h(k)+1}=a_{r+1}=1$.

Corollary 4.2 gives an alternative way of defining $h(k)$, in terms of RCF approximation constants $\Theta_{r}$, which leads to Selenius' algorithm.

LeMma 4.3. Let $h(k)=r$ and $\Theta_{r}=H\left(A_{r} / B_{r}\right)$.

(a) If $a_{r+1}>1$, then $\epsilon_{k+1}=1$ and $h(k+1)=h(k)+1$.

(b) If $a_{r+1}=1$; then:

(i) $\Theta_{r}<\Theta_{r+1}$ implies that $\epsilon_{k+1}=1$ and $h(k+1)=h(k)+1$;

(ii) $\Theta_{r}>\Theta_{r+1}$ implies that $\epsilon_{k+1}=-1$ and $h(k+1)=h(k)+2$.

Proof. The contrapositive of (a) follows, as $\epsilon_{k+1}=-1$ implies that $a_{h(k)+1}=a_{r+1}=1$, while (b)(i) and (ii) follow on taking contrapositives in Corollary 4.2(b) and (a).

Remark 4.4. In cases (a) and (b)(i), we represent the change in $h(k)$ as a jump of one RCF partial quotient:

$$
\vec{a}_{r}, a_{r+1}
$$

In case (b)(ii) we represent the change in $h(k)$ as a jump of two RCF partial quotients:

$$
\vec{a}_{r}, 1, a_{r+2}
$$


Lemma 4.3(b) tells us that the RCF to OCF transition depends on the relative sizes of the approximation constants $\Theta_{r}$ corresponding to a unisequence, that is, a sequence of consecutive partial quotients 1 . The dependence is described in [6, Lemma 4.1] as follows.

Lemma 4.5. Consider the $k$-unisequence $a_{r}, 1, \ldots, 1, a_{r+k+1}$, where $a_{r}>1$ if $r>0$ and $a_{r+k+1}>1$.

(a) If $k$ is odd, then

$$
\Theta_{r}>\Theta_{r+1}, \Theta_{r+2}>\Theta_{r+3}, \ldots, \Theta_{r+k-1}>\Theta_{r+k}
$$

(b) If $k=2 t$ where $t$ is even, then

$$
\begin{gathered}
\Theta_{r}>\Theta_{r+1}, \Theta_{r+2}>\Theta_{r+3}, \ldots, \Theta_{r+t-2}>\Theta_{r+t-1}, \\
\Theta_{r+t}<\Theta_{r+t+1}, \\
\Theta_{r+t+1}>\Theta_{r+t+2}, \Theta_{r+t+3}>\Theta_{r+t+4}, \ldots, \Theta_{r+2 t-1}>\Theta_{r+2 t} .
\end{gathered}
$$

(c) If $k=2 t$ where $t$ is odd, then

$$
\begin{gathered}
\Theta_{r}>\Theta_{r+1}, \Theta_{r+2}>\Theta_{r+3}, \ldots, \Theta_{r+t-3}>\Theta_{r+t-2}, \\
\Theta_{r+t}>\Theta_{r+t+1}, \Theta_{r+t+2}>\Theta_{r+t+3}, \ldots, \Theta_{r+2 t-1}>\Theta_{r+2 t} .
\end{gathered}
$$

Further, $\Theta_{r+t+1}<\Theta_{r+t+2}$ if $t \geq 3$.

In (c), Selenius called the $k$-unisequence indirect (ungerade) or direct (gerade), according to whether $\Theta_{r+t-1}<\Theta_{r+t}$ or $\Theta_{r+t-1}>\Theta_{r+t}$.

REMARK 4.6. From Lemmas 4.3 and 4.5, we have Selenius' recipe for the variation in $h(k)$ on meeting a $k$-unisequence.

(a) $k$ odd: there are $(k+1) / 2$ jumps of length 2 .

(b) $k=2 t$ where $t$ is even: there are $t / 2$ jumps of 2 , then a jump of length 1 , then $t / 2$ jumps of length 2 .

(c) $k=2 t$ where $t$ is odd.

(i) If $\Theta_{r+t-1}>\Theta_{r+t}$, there are $(t+1) / 2$ jumps of length 2, then a jump of length 1 , followed by $(t-1) / 2$ jumps of length 2 .

(ii) If $\Theta_{r+t-1}<\Theta_{r+t}$, there are $(t-1) / 2$ jumps of length 2 , then a jump of length 1 , followed by $(t+1) / 2$ jumps of length 2 .

For example:

$k=1:{\overrightarrow{a_{r}, 1, a_{r+2}}}_{\longrightarrow}$

$k=2: \overrightarrow{a_{r}, 1}, \overrightarrow{1,} a_{r+3}$ (indirect unisequence, $\Theta_{r}<\Theta_{r+1}$ );

$k=2: \overrightarrow{a_{r}, 1,1}, \vec{a}_{r+3}$ (direct unisequence, $\Theta_{r}>\Theta_{r+1}$ );

$k=3: \overrightarrow{a_{r}, 1,1}, \overrightarrow{1, a_{r+4}}$;

$k=4: \overrightarrow{a_{r}, 1,1}, \overrightarrow{1}, \overrightarrow{1, a_{r+5}}$; 
$k=5: \overrightarrow{a_{r}, 1,1,}, \overrightarrow{1,1}, \overrightarrow{1, a_{r+6}}$

$k=6$ :

(i) $\overrightarrow{a_{r}, 1,1}, \overrightarrow{1}, \overrightarrow{1,}, \overrightarrow{1,} a_{r+7}$ (indirect unisequence, $\Theta_{r+2}<\Theta_{r+3}$ );

(ii) $\overrightarrow{a_{r}, 1,1}, \overrightarrow{1,1}, \overrightarrow{1}, \overrightarrow{1, a_{r+7}}$ (direct unisequence, $\Theta_{r+2}>\Theta_{r+3}$ ).

REMARK 4.7. It is well known [4, p. 29] that

$$
\Theta_{i}=\left\{\left[a_{i+1}, \ldots, a_{1}\right]+\left[0, a_{i+2}, a_{i+3}, \ldots\right]\right\}^{-1} .
$$

As pointed out by Selenius [7], for a $k$-unisequence with $k=2 t$ and $t$ odd,

$$
\Theta_{r+t-1}>\Theta_{r+t} \Leftrightarrow\left[a_{r}, a_{r-1}, \ldots, a_{1}\right]>\left[a_{r+2 t+1}, a_{r+2 t+2}, \ldots\right]
$$

if $r>0$.

The following result from [3] is useful in establishing inequalities between regular continued fractions.

Lemma 4.8. Suppose that $x=\left[a_{0}, \ldots, a_{k-1}, a_{k}, \ldots\right], x^{\prime}=\left[a_{0}, \ldots, a_{k-1}, a_{k}^{\prime}, \ldots\right]$ with $a_{k} \neq a_{k}^{\prime}$. Then $a_{k}<a_{k}^{\prime}$ if and only if $k$ is even and $x<x^{\prime}$, or $k$ is odd and $x>x^{\prime}$.

There is one occasion when we always have an indirect unisequence.

LeMma 4.9. If $k=2 t$ where $t$ is odd, then the $k$-unisequence $a_{0},(1)^{k}, a_{2 t+1}$, where $a_{2 t+1}>1$, satisfies

$$
\Theta_{t-1}>\Theta_{t} .
$$

Proof. Using the notation of Selenius [7, p. 18], where $\gamma_{t}=\Theta_{t-1}^{-1}$, we prove equivalently that $\gamma_{t}<\gamma_{t+1}$.

$$
\begin{aligned}
\gamma_{t} & =\left[a_{t}, \ldots, a_{1}\right]+\left[0, a_{t+1}, \ldots\right] \\
& =\left[(1)^{t}\right]+\left[0,(1)^{t}, a_{2 t+1}, \ldots\right]=y+1 / x, \\
\gamma_{t+1} & =\left[(1)^{t+1}\right]+\left[0,(1)^{t-1}, a_{2 t+1}, \ldots\right]=1 / y+x,
\end{aligned}
$$

where $y=\left[(1)^{t}\right]$ and $x=\left[(1)^{t}, a_{2 t+1}, \ldots\right]$. Hence

$$
\begin{aligned}
\gamma_{t}<\gamma_{t+1} & \Longleftrightarrow y+\frac{1}{x}<\frac{1}{y}+x \\
& \Longleftrightarrow \frac{x y+1}{x}<\frac{1+x y}{y} \\
& \Longleftrightarrow y<x
\end{aligned}
$$

Now by [7, p. 30],

$$
y=\frac{F_{t}}{F_{t-1}}, \quad x=\frac{F_{t} \xi_{2 t+1}+F_{t-1}}{F_{t-1} \xi_{2 t+1}+F_{t-2}},
$$


where $F_{n}$ is the $n$th Fibonacci number (that is, $F_{-1}=0, F_{0}=1$, and $F_{n}=F_{n-1}+F_{n-2}$ when $n \geq 1)$ and $\xi_{2 t+1}=\left[a_{2 t+1}, \ldots\right]$. Then

$$
\begin{aligned}
y<x & \Longleftrightarrow \frac{F_{t}}{F_{t-1}}<\frac{F_{t} \xi_{2 t+1}+F_{t-1}}{F_{t-1} \xi_{2 t+1}+F_{t-2}} \\
& \Longleftrightarrow F_{t} F_{t-1} \xi_{2 t+1}+F_{t} F_{t-2}<F_{t-1} F_{t} \xi_{2 t+1}+F_{t-1}^{2} \\
& \Longleftrightarrow 0<F_{t-1}^{2}-F_{t} F_{t-2}=(-1)^{t-1}=1,
\end{aligned}
$$

as required.

For use in Section 8, we need the following result.

LEMMA 4.10. If OCF complete quotient $\tilde{\xi}_{m}=\left(p-1+\sqrt{p^{2}+1}\right) / 2$, where $p \geq 3$ and $m>0$, then:

(a) $\tilde{\xi}_{m+1}=\left(p-1+\sqrt{p^{2}+1}\right) / p$;

(b) $\tilde{\xi}_{m}$ starts an OCF period of length 4 .

Proof. (a) Suppose that $\tilde{\xi}_{m}=[\overline{p-1,1,1}]$. Then $\tilde{\xi}_{m}=\xi_{r}+1$ or $\xi_{r}$, where $\xi_{r}=$ $\left[a_{r}, a_{r+1}, \ldots\right]$. In the first case, $\xi_{r}=[p-2,1,1, p-1, \ldots]$ and $\epsilon_{m}=-1$. Then we have $\Theta_{r}<\Theta_{r+1}$, as

$$
\left[a_{r}, \ldots, a_{1}\right]=\left[p-2, \ldots, a_{1}\right]<\left[a_{r+3}, a_{r+4}, \ldots\right]=[p-1,1,1, \ldots],
$$

using (4.3) and Lemma 4.8. Hence $\epsilon_{m+1}=1$.

In the second case, $\xi_{r}=[\overline{p-1,1,1}]$ and $\epsilon_{m}=1$. The following cases are those that give $\epsilon_{m}=1$ :

(i) (a) $r=1: \overrightarrow{a_{0}, p-1 \text {; }}$

(b) $r>1: a_{r-1}>1: a_{0}, \ldots, \overrightarrow{a_{r-1}, \quad p-1}$;

(ii) (a) $r=3: \overrightarrow{a_{0}, 1,1}, \overrightarrow{p-1}$;

(b) $r>3: a_{r-3}>p-1$;

(c) $\quad a_{r-3}=p-1$ and $\Theta_{r-3}>\Theta_{r-2}: a_{0}, \ldots, \overrightarrow{a_{r-3}, 1,1,} \overrightarrow{p-1}$.

These all give $\epsilon_{m+1}=1$, using (4.3), Lemmas 4.8 and 4.9. Only (ii)(c) needs care; we assume that $\Theta_{r-3}>\Theta_{r-2}$. Then (4.3), with $D=p^{2}+1$, gives

$$
\theta=\left[a_{r-3}, a_{r-2}, \ldots, a_{1}\right]>\left[a_{r}, a_{r+1}, \ldots\right]=(p-1+\sqrt{D}) / 2 .
$$

We have to deduce $\Theta_{r}<\Theta_{r+1}$, that is,

$$
\left[a_{r}, a_{r-1}, \ldots, a_{1}\right]=[p-1,1,1, \theta]<\left[a_{r+3}, a_{r+4}, \ldots\right]=(p-1+\sqrt{D}) / 2 .
$$

Happily this turns out to be equivalent to inequality (4.4).

In view of $\epsilon_{m+1}=1$, we choose the positive representation in the equation

$$
\tilde{\xi}_{m}=(p-1+\sqrt{D}) / 2=p-1+\frac{p}{p-1+\sqrt{D}}=p-\frac{p}{p+1+\sqrt{D}},
$$

to get $\tilde{\xi}_{m+1}=(p-1+\sqrt{D}) / p$. 
(b) Periodicity. We have the positive-negative representation

$$
\tilde{\xi}_{m+1}=(p-1+\sqrt{D}) / p=1+\frac{p}{1+\sqrt{D}}=2-\frac{2}{p+1+\sqrt{D}} .
$$

Then $\epsilon_{m+2}=-1$, as $\tilde{a}_{m+1}>1$ by [2, Corollary 4.27] and hence

$$
\tilde{\xi}_{m+2}=(p+1+\sqrt{D}) / 2=p+\frac{p}{p-1+\sqrt{D}}=p+1-\frac{p}{p+1+\sqrt{D}} .
$$

Also $\epsilon_{m+1}=1$ implies that $\Theta_{r}<\Theta_{r+1}$ and using (4.3), we have $\Theta_{r+3}>\Theta_{r+4}$ and hence $\epsilon_{m+3}=-1$. Consequently,

$$
\tilde{\xi}_{m+3}=(p+1+\sqrt{D}) / p=2+\frac{2}{p-1+\sqrt{D}}=3-\frac{3 p-4}{2 p-1+\sqrt{D}} .
$$

Then, as $a_{r+3}=p-1>1$, by Lemma 4.3(a), we have $\epsilon_{m+4}=1$ and hence

$$
\tilde{\xi}_{m+4}=(p-1+\sqrt{D}) / 2=\tilde{\xi}_{m},
$$

thus establishing periodicity, with corresponding section of the OCF expansion

$$
\cdots \frac{\epsilon_{m} \mid}{\mid p-1}+\frac{1 \mid}{\mid 2}-\frac{1 \mid}{\mid p+1}-\frac{1 \mid}{\mid 2}+\cdots,
$$

as required.

\section{The NSCF algorithm}

To define the NSCF, we start with a quadratic irrationality $\xi_{0}=\left(P_{0}+\sqrt{D}\right) / Q_{0}$ in standard form, that is, $D$ is a nonsquare positive integer and $P_{0}, Q_{0},\left(D-P_{0}^{2}\right) / Q_{0}$ are integers, having no common factor other than 1 . We define complete quotients recursively. Suppose that $\tilde{\xi}_{0}=\xi_{0}$ and that $\tilde{\xi}_{m}=\left(\tilde{P}_{m}+\sqrt{D}\right) / \tilde{Q}_{m}$ is in standard form and let $c_{m}=\left\lfloor\tilde{\xi}_{m}\right\rfloor$. Then we have the positive and negative representations

$$
\tilde{\xi}_{m}=\left(\tilde{P}_{m}+\sqrt{D}\right) / \tilde{Q}_{m}=c_{m}+\frac{\tilde{Q}_{m+1}}{\tilde{P}_{m+1}+\sqrt{D}}=c_{m}+1-\frac{\tilde{Q}_{m+1}^{\prime \prime}}{\tilde{P}_{m+1}^{\prime \prime}+\sqrt{D}},
$$

where $\left(\tilde{P}_{m+1}+\sqrt{D}\right) / \tilde{Q}_{m+1}>1$ and $\left(\tilde{P}_{m+1}^{\prime \prime}+\sqrt{D}\right) / \tilde{Q}_{m+1}^{\prime \prime}>1$ are also in standard form. We choose

$$
\begin{aligned}
\tilde{a}_{m} & = \begin{cases}c_{m} & \text { if }\left|\tilde{Q}_{m+1}^{\prime}\right|<\left|\tilde{Q}_{m+1}^{\prime \prime}\right|, \text { or }\left|\tilde{Q}_{m+1}^{\prime}\right|=\left|\tilde{Q}_{m+1}^{\prime \prime}\right| \text { and } \tilde{Q}_{m}<0, \\
c_{m}+1 & \text { if }\left|\tilde{Q}_{m+1}^{\prime}\right|>\left|\tilde{Q}_{m+1}^{\prime \prime}\right|, \text { or }\left|\tilde{Q}_{m+1}^{\prime}\right|=\left|\tilde{Q}_{m+1}^{\prime \prime}\right| \text { and } \tilde{Q}_{m}>0,\end{cases} \\
\epsilon_{m+1} & =\operatorname{sign}\left(\tilde{\xi}_{m}-\tilde{a}_{m}\right), \\
\tilde{\xi}_{m+1} & = \begin{cases}\left(\tilde{P}_{m+1}^{\prime}+\sqrt{D}\right) / \tilde{Q}_{m+1}^{\prime} & \text { if } \epsilon_{m+1}=1, \\
\left(\tilde{P}_{m+1}^{\prime \prime}+\sqrt{D} / \tilde{Q}_{m+1}^{\prime \prime}\right. & \text { if } \epsilon_{m+1}=-1 .\end{cases}
\end{aligned}
$$


For $m \geq 0$, we also have

$$
\tilde{\xi}_{m}=\tilde{a}_{m}+\frac{\epsilon_{m+1}}{\tilde{\xi}_{m+1}} .
$$

If $\tilde{Q}_{m}, \tilde{Q}_{m+1}^{\prime}, \tilde{Q}_{m+1}^{\prime \prime}$ are all positive, we have the simplification

$$
\tilde{a}_{m}=\left\{\begin{array}{ll}
c_{m} & \text { if } \tilde{Q}_{m+1}^{\prime}<\tilde{Q}_{m+1}^{\prime \prime}, \\
c_{m}+1 & \text { if } \tilde{Q}_{m+1}^{\prime} \geq \tilde{Q}_{m+1}^{\prime \prime},
\end{array} \quad \epsilon_{m+1}= \begin{cases}1 \quad \text { if } \tilde{Q}_{m+1}^{\prime}<\tilde{Q}_{m+1}^{\prime \prime}, \\
-1 \quad \text { if } \tilde{Q}_{m+1}^{\prime} \geq \tilde{Q}_{m+1}^{\prime \prime}\end{cases}\right.
$$

This will be the case for all $m \geq n$ if $\tilde{\xi}_{n}$ is NSCF reduced, or if $\left|\tilde{Q}_{n}\right|<2 \sqrt{D}$ (see [1, Definition, p. 27] and [1, Theorem I, p. 22]). Such an index $n$ always exists. The NSCF expansion of a quadratic surd is ultimately periodic (see [1, Theorem II, p. 25]). This is equivalent to $\tilde{\xi}_{i_{0}}=\tilde{\xi}_{i_{0}+p}$, where $p>0$. The NSCF reduced surds are precisely the surds with a purely periodic NSCF expansion (see [1, pp. 101-102]).

Lemma 5.1 (John Robertson). If $\xi>2$ is RCF reduced, then $\xi$ or $\xi+1$ is NSCF reduced.

Proof. This depends on the fact that if $\xi$ is NSCF reduced, then $\xi$ or $\xi-1$ is RCF reduced (see [6, Lemma (6.2)]). So a cycle $\left\{\xi_{i}\right\}$ of NSCF reduced surds corresponds to a subsequence $\left\{\eta_{\phi(i)}\right\}$ of a cycle $\left\{\eta_{j}\right\}$ of RCF reduced surds. If $p$ is the RCF cycle length, we see by Lemma 3.4 that $\phi(i+1)=\phi(i)+1$ or $\phi(i)+2$ for $i$ (modulo $p$ ) and in the latter case $a_{\phi(i)+1}=1$, which implies that $\eta_{\phi(i)+1}=1+1 / \eta_{\phi(i)+2}<2$.

Corollary 5.2. Assume that $\tilde{\xi}_{k}$ is an OCF complete quotient. If $a_{h(k)}>1$ and $\xi_{h(k)}$ is $R C F$ reduced, then one of $\tilde{\xi}_{k}$ or $\tilde{\xi}_{k} \pm 1$ is NSCF reduced.

Proof. If $\tilde{\xi}_{k}$ is an OCF complete quotient, then by (3.3) $\tilde{\xi}_{k}=\xi_{h(k)}$ or $\xi_{h(k)}+1$. Then $a_{h(k)}>1$ implies that $\xi_{h(k)}>2$, so if $\xi_{h(k)}$ is RCF reduced, Lemma 5.1 implies that $\xi_{h(k)}$ or $\xi_{h(k)}+1$ is NSCF reduced. Hence one of $\tilde{\xi}_{k}, \tilde{\xi}_{k} \pm 1$ is NSCF reduced.

Corollary 5.3. There exists a OCF complete quotient $\tilde{\xi}_{k}$, such that $\tilde{\xi}_{k}$ or $\tilde{\xi}_{k} \pm 1$ is NSCF reduced.

Proof. If $\xi_{0}$ is equivalent to $(1+\sqrt{5}) / 2$, then $\xi_{n}=(1+\sqrt{5}) / 2$ for all $n \geq n_{0}$ and so $\tilde{\xi}_{k}=(3+\sqrt{5}) / 2$ for all $k$ such that $h(k) \geq n_{0}$. Also $(3+\sqrt{5}) / 2$ is NSCF reduced. Otherwise there exists $n$ such that $a_{n}>1$ and hence, by Lemma 3.5, $h(k)=n$ for some $k$. If we choose $n$ large enough so that $\xi_{n}$ is RCF reduced, then Corollary 5.2 gives the desired result.

\section{Some inequalities of Selenius}

In this section, we establish a connection between the OCF and NSCF algorithms, based on a result of Selenius.

Lemma 6.1 [7, Satz 29]. Suppose that $\Theta_{r}=B_{r}\left|B_{r} \xi_{0}-A_{r}\right|$, where $A_{r} / B_{r}$ is the rth $R C F$ convergent to $\xi_{0}=\left(P_{0}+\sqrt{D}\right) / Q_{0}$. Suppose that $Q_{r}$ and $Q_{r+1}$ are positive for all large $r \geq 0$. 
(a) If $Q_{r+1}=Q_{r}$, then for $r \geq 1$,

$$
(-1)^{r}\left(\Theta_{r}-\Theta_{r-1}\right) t>0
$$

where $t=\operatorname{sign}\left(Q_{0}\right)$.

(b) If $r$ is sufficiently large (for example, $B_{r} B_{r+1}>\left|Q_{0}\right|$ ) and $Q_{r+1} \neq Q_{r}$, then

$$
Q_{r+1}<Q_{r} \Longleftrightarrow \Theta_{r}<\Theta_{r-1}
$$

Moreover, if $\xi_{0}=\sqrt{D}$, then (6.1) holds for $r \geq 1$.

(Selenius stated his result in terms of $\gamma_{r}=1 / \Theta_{r-1}$.)

Corollary 6.2. Assume that $\xi_{h(k)}$ is RCF reduced and $\tilde{\xi}_{k}$ has positive-negative representation

$$
\tilde{\xi}_{k}=\left(\tilde{P}_{k}+\sqrt{D}\right) / \tilde{Q}_{k}=b_{k}+\frac{\tilde{Q}_{k+1}^{\prime}}{\tilde{P}_{k+1}+\sqrt{D}}=b_{k}+1-\frac{\tilde{Q}_{k+1}^{\prime \prime}}{\tilde{P}_{k+1}^{\prime \prime}+\sqrt{D}} .
$$

(a) Suppose that $\tilde{Q}_{k+1}^{\prime}=\tilde{Q}_{k+1}^{\prime \prime}$. Then

$$
H\left(\frac{u_{k}}{v_{k}}\right)>H\left(\frac{u_{k}+r_{k-1}}{v_{k}+s_{k-1}}\right) \Longleftrightarrow(-1)^{k} \epsilon_{1} \cdots \epsilon_{k} t=1,
$$

where $t=\operatorname{sign}\left(Q_{0}\right)$.

(b) Suppose that $\tilde{Q}_{k+1}^{\prime} \neq \tilde{Q}_{k+1}^{\prime \prime}$ and $a_{h(k)+1}=1$. Then if $s_{k}>\left|Q_{0}\right|$,

$$
H\left(\frac{u_{k}}{v_{k}}\right)>H\left(\frac{u_{k}+r_{k-1}}{v_{k}+s_{k-1}}\right) \Longleftrightarrow \tilde{Q}_{k+1}^{\prime}>\tilde{Q}_{k+1}^{\prime \prime}
$$

REMARK 6.3. If $\xi_{0}=\sqrt{D}$, then (b) holds for all $k \geq 0$.

Proof. From (3.6), $\tilde{Q}_{k+1}^{\prime}=Q_{h(k)+1}$ and $\tilde{Q}_{k+1}^{\prime \prime}=Q_{h(k)+1}^{\prime \prime}$ and both are positive if $\xi_{h(k)}$ is RCF reduced, by [1, Theorem I, p. 22], as then $0<Q_{h(k)}<2 \sqrt{D}$.

For case (a), $\tilde{Q}_{k+1}^{\prime}=\tilde{Q}_{k+1}^{\prime \prime}$ becomes $Q_{h(k)+1}=Q_{h(k)+1}^{\prime \prime}$. Then $a_{h(k)+1}=1$, by Lemma 3.4, and hence $Q_{h(k)+1}=Q_{h(k)+1}^{\prime \prime}=Q_{h(k)+2}$. Lemma 6.1(a), with $r=h(k)+1$, now gives

$$
(-1)^{h(k)+1}\left(H\left(\frac{A_{h(k)+1}}{B_{h(k)+1}}\right)-H\left(\frac{A_{h(k)}}{B_{h(k)}}\right)\right) t>0
$$

and Lemma 4.1 gives

$$
(-1)^{h(k)+1}\left(H\left(\frac{u_{k}+r_{k-1}}{v_{k}+s_{k-1}}\right)-H\left(\frac{u_{k}}{v_{k}}\right)\right) t>0 .
$$


Also

$$
\begin{aligned}
(-1)^{h(k)+1} & =A_{h(k)} B_{h(k)+1}-A_{h(k)+1} B_{h(k)} \\
& =\left(v_{k}+s_{k-1}\right) u_{k}-v_{k}\left(u_{k}+r_{k-1}\right) \\
& =u_{k} s_{k-1}-v_{k} r_{k-1} \\
& =\left(b_{k} r_{k-1}+\epsilon_{k} r_{k-2}\right) s_{k-1}-\left(b_{k} s_{k-1}+\epsilon_{k} s_{k-2}\right) r_{k-1} \\
& =\epsilon_{k}\left(r_{k-2} s_{k-1}-r_{k-1} s_{k-2}\right) \\
& =(-1)^{k+1} \epsilon_{1} \cdots \epsilon_{k} .
\end{aligned}
$$

So inequality (6.3) gives

$$
(-1)^{k+1} \epsilon_{1} \cdots \epsilon_{k}\left(H\left(\frac{u_{k}+r_{k-1}}{v_{k}+s_{k-1}}\right)-H\left(\frac{u_{k}}{v_{k}}\right)\right) t>0
$$

and this gives (6.2).

For case (b), if $a_{h(k)+1}=1$, we again have $Q_{h(k)+1}^{\prime \prime}=Q_{h(k)+2}$, so Lemma 6.1(b) applies with $r=h(k)+1$. We also note that

$$
B_{r} B_{r+1}=v_{k}\left(v_{k}+s_{k-1}\right)= \begin{cases}s_{k}\left(s_{k}+s_{k-1}\right) & \text { if } \epsilon_{k+1}=1, \\ \left(s_{k}-s_{k-1}\right) s_{k} & \text { if } \epsilon_{k+1}=-1 .\end{cases}
$$

So $B_{r} B_{r+1}>\left|Q_{0}\right|$ if $s_{k}>\left|Q_{0}\right|$.

The case where $a_{h(k)+1}>1$ is easier to deal with.

LEMMA 6.4. If $\xi_{h(k)}$ is $R C F$ reduced and $a_{h(k)+1}>1$, then:

(i) $H\left(u_{k} / v_{k}\right)<H\left(\left(u_{k}+r_{k-1}\right) /\left(v_{k}+s_{k-1}\right)\right)$;

(ii) $\tilde{Q}_{k+1}^{\prime}<\tilde{Q}_{k+1}^{\prime \prime}$.

Proof. Assume that $a_{h(k)+1}>1$. Then we know that $\epsilon_{k+1}=1$ and hence (i) holds.

If $\tilde{Q}_{k+1}^{\prime} \geq \tilde{Q}_{k+1}^{\prime \prime}$, then $Q_{h(k)+1} \geq Q_{h(k)+1}^{\prime \prime}$ and so $a_{h(k)+1}=1$, by Lemma 3.4(b)(ii).

The phenomenon $\tilde{Q}_{m+1}^{\prime}=\tilde{Q}_{m+1}^{\prime \prime}$ is now investigated in detail.

Lemma 6.5. Suppose that $\tilde{\xi}_{m}$ is an OCF complete quotient for which $\tilde{\xi}_{m}+b$ is also $N S C F$ reduced for some integer $b$. If $\tilde{Q}_{m+1}^{\prime}=\tilde{Q}_{m+1}^{\prime \prime}$, then

$$
\tilde{\xi}_{m}=\left((2 a+1) q-p+\sqrt{p^{2}+q^{2}}\right) / 2 q, \quad p \geq 2 q>0,
$$

where $a=\left\lfloor\tilde{\xi}_{m}\right\rfloor$.

Proof. Suppose that $\tilde{Q}_{m+1}^{\prime}=\tilde{Q}_{m+1}^{\prime \prime}$. Then from [1, Theorem 1, p. 22],

$$
\begin{aligned}
\tilde{P}_{m+1}^{\prime \prime}+\tilde{P}_{m+1}^{\prime} & =2 \tilde{Q}_{m+1}^{\prime \prime} \\
\tilde{P}_{m+1}^{\prime \prime}-\tilde{P}_{m+1}^{\prime} & =\tilde{Q}_{m} \\
2 \tilde{P}_{m+1}^{\prime \prime} & =\tilde{Q}_{m}+2 \tilde{Q}_{m+1}^{\prime \prime} \\
\tilde{P}_{m+1}^{\prime \prime} & =\frac{1}{2} \tilde{Q}_{m}+\tilde{Q}_{m+1}^{\prime \prime}
\end{aligned}
$$


Also

$$
\begin{aligned}
D & =\left(\tilde{P}_{m+1}^{\prime \prime}\right)^{2}-\tilde{Q}_{m} \tilde{Q}_{m+1}^{\prime \prime} \\
& =\left(\frac{1}{2} \tilde{Q}_{m}+\tilde{Q}_{m+1}^{\prime \prime}\right)^{2}-\tilde{Q}_{m} \tilde{Q}_{m+1}^{\prime \prime} \\
& =\left(\frac{1}{2} \tilde{Q}_{m}\right)^{2}+\left(\tilde{Q}_{m+1}^{\prime \prime}\right)^{2} \\
& =q^{2}+p^{2},
\end{aligned}
$$

where $p=Q_{m+1}^{\prime \prime}$ and $q=\frac{1}{2} \tilde{Q}_{m}$, where $p>0$, since $0<\tilde{Q}_{m}<\sqrt{D}$, by $\quad[1$, Theorem 1(iv), p. 22] and $q>0$. Again, by [1, p. 22],

$$
\tilde{P}_{m}=a \tilde{Q}_{m}-\tilde{P}_{m+1}^{\prime}=2 a q-(p-q)=(2 a+1) q-p .
$$

Hence

$$
\tilde{\xi}_{m}=\left(\tilde{P}_{m}+\sqrt{p^{2}+q^{2}}\right) / \tilde{Q}_{m}=\left((2 a+1) q-p+\sqrt{p^{2}+q^{2}}\right) / 2 q .
$$

Hence $\tilde{Q}_{m+1}=p$. Also $\tilde{\xi}_{m}+b=\left(\tilde{P}_{m}+b \tilde{Q}_{m}+\sqrt{D}\right) / \tilde{Q}_{m}$ is a special NSCF complete quotient ([1, Theorem IV, p. 28]), whose NSCF successor is $\left(p+q+\sqrt{p^{2}+q^{2}} / p\right.$, so

$$
\tilde{Q}_{m}^{2}+\frac{1}{4} p^{2} \leq D
$$

Hence (6.4) gives $4 q^{2}+\frac{1}{4} p^{2} \leq p^{2}+q^{2}$ and so $2 q \leq p$.

\section{Occurrences of $\left(p+q+\sqrt{p^{2}+q^{2}}\right) / p, p>2 q>0$ in an NSCF period}

Ayyangar proved that there are at most two complete quotients of the form $(p+q+$ $\left.\sqrt{p^{2}+q^{2}}\right) / p$, where $p>2 q>0$, in an NSCF period (see [1, Lemma (2), p. 106]). The case of just one occurrence was described by Ayyangar [1, 5.6.2, pp. 112-113]. In the case where there are two occurrences, we need to know that they are separated by half a period length. This is stated explicitly in the next result. We will also need part (d) in the proof of Theorem 8.1.

LEMMA 7.1. Let $k$ be the period length of the NSCF expansion of $\left(p+q+\sqrt{p^{2}+q^{2}}\right) / p$, where $p>2 q>0$. If some $\tilde{\xi}_{h}$, where $1 \leq h<k$, also has this form, then:

(a) $k$ is even and $h=k / 2$;

(b) $\tilde{Q}_{v}=\tilde{Q}_{k-v}$ for $0 \leq v \leq k-1$;

(c) $\tilde{P}_{k+1-v}=\tilde{P}_{v}$ for $2 \leq v \leq \frac{1}{2} k-1$;

(d) $\tilde{a}_{v}=\tilde{a}_{k-v}, 2 \leq v \leq \frac{1}{2} k-2, \tilde{a}_{k-1}-1=\tilde{a}_{1}, \tilde{a}_{k / 2+1}=\tilde{a}_{k / 2-1}-1, \tilde{a}_{k / 2}=2$;

(e) $\epsilon_{k+1-v}=\epsilon_{v}, 2 \leq v \leq \frac{1}{2} k-1, \epsilon_{k / 2}=-1, \epsilon_{k / 2+1}=1, \epsilon_{1}=1, \epsilon_{k}=-1$.

Proof. See [5] for a proof that is an extension of the argument in [1, pp. 112-113], which dealt with the case where there is only one complete quotient of the above type in a period.

Example 7.2 (see Table 3 ). Here $\xi_{0}=(324+\sqrt{81770}) / 283=\left(p+q+\sqrt{p^{2}+q^{2}}\right) / p$, where $p>2 q>0$. Then $\tilde{\xi}_{4}=(348+\sqrt{81770}) / 277=\left(P+Q+\sqrt{P^{2}+Q^{2}}\right) / p$, where $P>2 Q>0$. Here $p^{2}+q^{2}=P^{2}+Q^{2}=81770$, where $p=283, q=41, P=277$ and $Q=71$. Also $k=8$ and $h=4$. The two occurrences are highlighted. 
TABLE 3. NSCF expansion of $(324+\sqrt{81770}) / 283$

\begin{tabular}{rcrccc}
\hline$i$ & $\tilde{\xi}_{i}$ & $\epsilon_{i}$ & $\tilde{a}_{i}$ & $\tilde{Q}_{i+1}^{\prime}$ & $\tilde{Q}_{i+1}^{\prime \prime}$ \\
\hline 0 & $(324+\sqrt{81770}) / 283$ & 1 & 2 & 82 & 685 \\
1 & $(242+\sqrt{81770}) / 82$ & 1 & 6 & 235 & 347 \\
2 & $(250+\sqrt{81770}) / 235$ & 1 & 2 & 142 & 533 \\
3 & $(220+\sqrt{81770}) / 142$ & -1 & 4 & $\mathbf{2 7 7}$ & $\mathbf{2 7 7}$ \\
4 & $(348+\sqrt{81770}) / 277$ & 1 & 2 & 142 & 547 \\
5 & $(206+\sqrt{81770}) / 142$ & 1 & 3 & 235 & 347 \\
6 & $(220+\sqrt{81770}) / 235$ & 1 & 2 & 82 & 653 \\
7 & $(250+\sqrt{81770}) / 82$ & -1 & 7 & $\mathbf{2 8 3}$ & $\mathbf{2 8 3}$ \\
8 & $(324+\sqrt{81770}) / 283$ & 1 & 2 & 82 & 685 \\
\hline
\end{tabular}

\section{The structure of the OCF period of a general quadratic surd}

Selenius proved periodicity with possible doubling for the SK expansion of $\sqrt{D}$ and stated without proof that for a general quadratic surd, the SK algorithm is periodic and that the period-doubling phenomenon can occur (see [7, Satz 36]). This was proved by Bosma and Kraaikamp [3, Theorem (6.1)]. We prove periodicity of the OCF algorithm by showing how the OCF period is related to the NSCF period. We have the following possible OCF periods.

THEOREM 8.1.

(a) If no complete quotient in an NSCF period of $\xi_{0}$ has the form $(p+q+$ $\left.\sqrt{p^{2}+q^{2}}\right) / p$, where $p>2 q>0$, for example, if a period contains only $m$ unisequences with $m$ odd or $m \equiv 0(\bmod 4)$, or if $\xi_{0}$ is equivalent to $(1+\sqrt{5}) / 2$, then the OCF and NSCF ultimately agree.

(b) Suppose there is only one occurrence in an NSCF period. We write L-RCF, $\mathrm{L}-\mathrm{NSCF}$, and L-OCF to denote the RCF, NSCF and OCF period lengths for $\xi_{0}$. Then:

(i) $\mathrm{L}-\mathrm{OCF}=2 \mathrm{~L}-\mathrm{NSCF}$ if $\mathrm{L}-\mathrm{RCF}$ is odd. This gives a triad reversal if $\mathrm{L}-\mathrm{NSCF} \geq 3$, while if $\mathrm{L-NSCF}=2$, then $D=p^{2}+1, p \geq 3$ and the OCF and NSCF periods have the form

$$
\mathrm{NSCF}: p_{*}-\frac{1 \mid}{\mid 2}+\cdots, \quad \text { OCF }: p-1+\frac{1 \mid}{\mid 2}-\frac{1 \mid}{\mid p+1}-\frac{1 \mid}{\mid 2}+\cdots .
$$

(ii) $\mathrm{L}-\mathrm{OCF}=\mathrm{L}-\mathrm{NSCF}$ if $\mathrm{L}-\mathrm{RCF}$ is even. The OCF period is essentially the same as the NSCF period, apart from a possible triad reversal.

(c) If we have two complete quotients of the form $\left(p+q+\sqrt{p^{2}+q^{2}}\right) / p$, where $p>2 q>0$ in the NSCF period of $\xi_{0}$, the periods of the OCF and NSCF will 
be essentially the same, apart from at most two reversals of triads of partial quotients in an NSCF period, corresponding to these two complete quotients.

EXAMPLE 8.2. Here are some examples of the various possible OCF period structures. For brevity, we define a special surd to be a quadratic irrationality of the form $\left(p+q+\sqrt{p^{2}+q^{2}}\right) / p$, where $p>2 q>0$.

(a) $\xi_{0}=(54+\sqrt{1584}) / 36$. Here no complete quotient in the NSCF period is a special surd.

$$
\begin{aligned}
& \text { NSCF: } 2+\frac{1 \mid}{\mid 2}-\frac{1 \mid}{\mid 3}-\frac{1 \mid}{\mid 8}-\frac{1 \mid}{\mid 3}-\frac{1 \mid}{\mid 3}-\frac{1 \mid}{\mid 3}-\frac{1 \mid}{\mid 3}+\cdots \\
& \mathrm{OCF}: 3-\frac{1 \mid}{\mid 2}+\frac{1 \mid}{\mid 2}-\frac{1 \mid}{\mid 8}-\frac{1 \mid}{\mid 3}-\frac{1 \mid}{\mid 3}-\frac{1 \mid}{\mid 3}-\frac{1 \mid}{\mid 3}+\cdots
\end{aligned}
$$

(b) In the following five examples, one complete quotient in the NSCF period is a special surd.

(i) $k^{\prime}=5, \mathrm{~L}-\mathrm{RCF}=9, \xi_{0}=(13+\sqrt{697}) / 22($ period doubling, one reversal).

$$
\begin{aligned}
& \mathrm{NSCF}: 2-\frac{1 \mid}{\mid 5}-\frac{1 \mid}{\mid 5}-\frac{1 \mid}{\mid 3}-\frac{1 \mid}{\mid 2}+\cdots \\
& \mathrm{OCF}: 2-\frac{1 \mid}{\mid 5}-\frac{1 \mid}{\mid 5}-\frac{1 \mid}{\mid 3}-\frac{1 \mid}{\mid 2}+\frac{1 \mid}{\mid 2}-\frac{1 \mid}{\mid 5}-\frac{1 \mid}{\mid 5}-\frac{1 \mid}{\mid 2}+\frac{1 \mid}{\mid 2}-\frac{1 \mid}{\mid 3}-\cdots .
\end{aligned}
$$

(ii) $k^{\prime}=4, \xi_{0}=\sqrt{29}$.

$$
\begin{aligned}
& \text { NSCF }: 5+\frac{1 \mid}{\mid 3}-\frac{1 \mid}{\mid 2}+\frac{1 \mid}{\mid 2}+\frac{1 \mid}{\mid 10}+\cdots \\
& \text { OCF: } 5+\frac{1 \mid}{\mid 2}+\frac{1 \mid}{\mid 2}-\frac{1 \mid}{\mid 3}+\frac{1 \mid}{\mid 10}+\frac{1 \mid}{\mid 3}-\frac{1 \mid}{\mid 2}+\frac{1 \mid}{\mid 2}+\frac{1 \mid}{\mid 10} \cdots
\end{aligned}
$$

(iii) $k^{\prime}=2, \xi_{0}=\frac{1}{2} \sqrt{(2 n+1)^{2}+1}$ where $n \geq 1$.

$$
\begin{aligned}
& \text { NSCF }: n+1-\frac{1 \mid}{\mid 2}+\frac{1 \mid}{\mid 2 n+1}-\cdots \\
& \text { OCF: } n+1-\frac{1 \mid}{\mid 2}+\frac{1 \mid}{\mid 2 n}+\frac{1 \mid}{\mid 2}-\frac{1 \mid}{\mid 2 n+2}-\cdots
\end{aligned}
$$

(iv) $k^{\prime}=9, \mathrm{~L}-\mathrm{RCF}=12, \xi_{0}=(19+\sqrt{205}) / 13$ (no reversal). 
(v) $k^{\prime}=3, \mathrm{~L}-\mathrm{RCF}=4, \xi_{0}=(2865+\sqrt{725}) / 1250$ (one reversal).

$$
\begin{aligned}
& \text { NSCF }: 2+\frac{1 \mid}{\mid 3}+\frac{1 \mid}{\mid 5}+\frac{1 \mid}{\mid 4}-\frac{1 \mid}{\mid 2}+\frac{1 \mid}{\mid 3}+\cdots \\
& \text { OCF }: 2+\frac{1 \mid}{\mid 3}+\frac{1 \mid}{\mid 5}+\frac{1 \mid}{\mid 3}+\frac{1 \mid}{\mid 2}-\frac{1 \mid}{\mid 4}+\cdots .
\end{aligned}
$$

(c) In the following three examples, two complete quotients in the NSCF period are special surds.

(i) $\quad \xi_{0}=(487+\sqrt{159197}) / 386:$ (no reversals)

$$
\mathrm{OCF}=\mathrm{NSCF}:{ }_{*}^{2}+\frac{1 \mid}{\mid 3}+\frac{1 \mid}{\mid 3}-\frac{1 \mid}{\mid 2}+\frac{1 \mid}{\mid 2}+\frac{1 \mid}{\mid 4}-\cdots .
$$

(ii) $\xi_{0}=(109+\sqrt{7453}) / 82:($ one reversal)

$$
\begin{aligned}
& \text { NSCF }: 2_{*}^{2}+\frac{1 \mid}{\mid 3}-\frac{1 \mid}{\mid 3}+\frac{1 \mid}{\mid 2}-\frac{1 \mid}{\mid 2}-\frac{1 \mid}{\mid 4}-\cdots \\
& \mathrm{OCF}: 2_{*}+\frac{1 \mid}{\mid 3}-\frac{1 \mid}{\mid 2}+\frac{1 \mid}{\mid 2}-\frac{1 \mid}{\mid 3}-\frac{1 \mid}{\mid 4}-\cdots .
\end{aligned}
$$

(iii) $\xi_{0}=(-41+\sqrt{1961}) / 28$ : (two reversals)

$$
\begin{aligned}
\text { NSCF: } \quad & +\frac{1 \mid}{\mid 9}-\frac{1 \mid}{\mid 2}+\frac{1 \mid}{\mid 8}+\frac{1 \mid}{\mid 3}+\frac{1 \mid}{\mid 22}-\frac{1 \mid}{\mid 6}-\frac{1 \mid}{\mid 2}+\frac{1 \mid}{\mid 3}-\frac{1 \mid}{\mid 3} \\
& -\frac{1 \mid}{\mid 2}+\frac{1 \mid}{\mid 2}-\frac{1 \mid}{\mid 3}+\frac{1 \mid}{\mid 2}-\frac{1 \mid}{\mid 6}-\frac{1 \mid}{\mid 22}+\frac{1 \mid}{\mid 3}+\cdots \\
\text { OCF: } \quad 0 & +\frac{1 \mid}{\mid 8}+\frac{1 \mid}{\mid 2}-\frac{1 \mid}{\mid 9}+\frac{1 \mid}{\mid 3}+\frac{1 \mid}{\mid 22}-\frac{1 \mid}{\mid 6}-\frac{1 \mid}{\mid 2}+\frac{1 \mid}{\mid 3}-\frac{1 \mid}{\mid 2} \\
& +\frac{1 \mid}{\mid 2}-\frac{1 \mid}{\mid 3}-\frac{1 \mid}{\mid 3}+\frac{1 \mid}{\mid 2}-\frac{1 \mid}{\mid 6}-\frac{1 \mid}{\mid 22}+\frac{1 \mid}{\mid 3}+\cdots .
\end{aligned}
$$

We need two preliminary results for the proof of Theorem 8.1.

LEMma 8.3. There exists an OCF complete quotient $\tilde{\xi}_{m}$ such that $\tilde{\xi}_{m}$ is NSCF reduced.

Proof. By Corollary 5.3, there exists $m$ such that $\tilde{\xi}_{m}$ or $\tilde{\xi}_{m} \pm 1$ is NSCF reduced and we can assume that $s_{m}>\left|Q_{0}\right|$ of Lemma 6.2(b) is satisfied and that $\xi_{h(m)}$ is RCF reduced. If $\tilde{Q}_{m+1}^{\prime} \neq \tilde{Q}_{m+1}^{\prime \prime}$, then the OCF and NSCF make the same choice and $\tilde{\xi}_{m+1}$ is NSCF reduced.

Otherwise we have $\tilde{Q}_{m+1}^{\prime}=\tilde{Q}_{m+1}^{\prime \prime}$ and hence by Lemma 6.5, with $D=p^{2}+q^{2}$,

$$
\tilde{\xi}_{m}=((2 a+1) q-p+\sqrt{D}) / 2 q=a+\frac{p}{p-q+\sqrt{D}}=a+1-\frac{p}{p+q+\sqrt{D}},
$$


where $p \geq 2 q>0$. Then $\tilde{\xi}_{m+1}=(p-q+\sqrt{D}) / p$ or $(p+q+\sqrt{D}) / p$. If $p=2 q$, then $p=2, q=1$ and $\tilde{\xi}_{m+1}=(3+\sqrt{5}) / 2$, as we know that $\tilde{\xi}_{m+1}>(1+\sqrt{5}) / 2$. Then $(3+$ $\sqrt{5}) / 2$ is NSCF reduced.

Now assume that $p>2 q$. If $\epsilon_{m+1}=-1$, then $\tilde{\xi}_{m+1}=(p+q+\sqrt{D}) / p$, which is NSCF reduced, whereas if $\epsilon_{m+1}=1$, then

$$
\tilde{\xi}_{m+1}=(p-q+\sqrt{D}) / p=1+\frac{p}{q+\sqrt{D}}=2-\frac{2 q}{p+q+\sqrt{D}} .
$$

Hence $\tilde{\xi}_{m+2}=(p+q+\sqrt{D}) / 2 q$, as we know that $\tilde{a}_{m+1} \geq 2$. Then $\tilde{\xi}_{m+2}=\eta+1$ where $\eta=(p-q+\sqrt{D}) / 2 q$ is NSCF reduced, as it is the NSCF successor of $(p+q+\sqrt{D}) / p$, by virtue of the positive-negative representation

$$
(p+q+\sqrt{D}) / p=2+\frac{2 q}{p-q+\sqrt{D}}=3-\frac{3 p-4 q}{2 p-q+\sqrt{D}}
$$

and the inequality $2 q<3 p-4 q$. If $\tilde{Q}_{m+3}^{\prime} \neq \tilde{Q}_{m+1}^{\prime \prime}$, then $\tilde{\xi}_{m+3}$ is the NSCF successor of $\eta$. Otherwise $\left(p-q+\sqrt{p^{2}+q^{2}}\right) / 2 q$ has the form $\left.(2 A+1) Q-P+\sqrt{P^{2}+Q^{2}}\right) / 2 Q$, where $P \geq 2 Q>0$. Then $q=Q, p=P$ and $p-q=(2 A+1) Q-P$. Hence $p=A+1$, $q=1$ and $p \geq 3$. Hence $\tilde{\xi}_{m+3}=\left(p+1+\sqrt{p^{2}+1}\right) / 2$ and by Lemma 4.10 ,

$$
\tilde{\xi}_{m+4}=\left(p+1+\sqrt{p^{2}+1}\right) / p,
$$

which is NSCF reduced.

LeMma 8.4. Suppose that $\tilde{\xi}_{m}$ is an OCF complete quotient that is NSCF reduced. Then $\xi_{h(m)}$ is RCF reduced if and only if $0<\tilde{P}_{m}<\sqrt{D}$ when $\epsilon_{m}=1$, while $\tilde{P}_{m}>\sqrt{D}$ when $\epsilon_{m}=-1$.

Proof. We know that $\tilde{\xi}_{m}=\xi_{h(m)}$ or $\xi_{h(m)}+1$, according to whether $\epsilon_{m}=1$ or -1 . Also if $\tilde{\xi}_{m}$ is NSCF reduced, then $-1<\left(\tilde{P}_{m}-\sqrt{D}\right) / \tilde{Q}_{m}<1$ (see [1, Theorem III, p. 27]). Further, $0<\tilde{Q}_{m}<\sqrt{D}$ (see [1, Theorem IV, p. 28]) and the definition of special surd at [1, p. 27]. Then $0<\tilde{P}_{m}$ follows from Theorem I(iv), [1, p. 22].

Case 1. Assume that $\epsilon_{m}=1$. Then $\tilde{P}_{m}<\sqrt{D}$ implies that $-1<\left(\tilde{P}_{m}-\sqrt{D}\right) / \tilde{Q}_{m}<0$, that is, $-1<\overline{\xi_{h(m)}}<0$; also $\xi_{h(m)}>1$ and hence $\xi_{h(m)}$ is RCF reduced. Also $\tilde{P}_{m}>\sqrt{D}$ implies that $0<\left(\tilde{P}_{m}-\sqrt{D}\right) / \tilde{Q}_{m}=\overline{\xi_{h(m)}}$, so $\xi_{h(m)}$ is not RCF reduced.

Case 2. Assume that $\epsilon_{m}=-1$. Then $\tilde{P}_{m}>\sqrt{D}$ implies that $0<\left(\tilde{P}_{m}-\sqrt{D}\right) / \tilde{Q}_{m}<1$, that is, $-1<\overline{\xi_{h(m)}}<0$; also $\xi_{h(m)}>1$ and hence $\xi_{h(m)}$ is RCF reduced. Also $\tilde{P}_{m}<\sqrt{D}$ implies that $\left(\tilde{P}_{m}-\sqrt{D}\right) / \tilde{Q}_{m}<0$ and hence $\left(\tilde{P}_{m}-\sqrt{D}\right) / \tilde{Q}_{m}-1=\overline{\xi_{h(m)}}<-1$. Hence $\xi_{h(m)}$ is not RCF reduced.

We now prove Theorem 8.1. We need the fact that equivalent quadratic surds eventually have the same NSCF expansions. This can be shown to be a consequence of the corresponding result for the RCF expansions. 
Proof. Find the first OCF complete quotient $\tilde{\xi}_{m}$ that is NSCF reduced and that satisfies $s_{m}>\left|Q_{0}\right|$ and either $\epsilon_{m}=1$ and $P_{m}<\sqrt{D}$ or $\epsilon_{m}=-1$ and $P_{m}>\sqrt{D}$. We show that $\tilde{\xi}_{m}$ starts an OCF period of length $k^{\prime}$ or $2 k^{\prime}$.

If $\tilde{Q}_{u+1}^{\prime} \neq \tilde{Q}_{u+1}^{\prime \prime}$ holds for all OCF $\tilde{\xi}_{u}$ in the range $m \leq u \leq m+k^{\prime}-1$, then the OCF and NSCF expansions will be identical for $u \geq m+1$ and $\tilde{\xi}_{m}$ starts an OCF period of length $k^{\prime}$.

Otherwise choose the first $u$ with $\tilde{Q}_{u+1}^{\prime}=\tilde{Q}_{u+1}^{\prime \prime}$, where $m \leq u \leq m+k^{\prime}-1$. Then as the OCF and NSCF make the same choice for $\tilde{\xi}_{m}, \ldots, \tilde{\xi}_{u-1}$, it follows that $\tilde{\xi}_{u}=\tilde{\eta}_{0}$ is NSCF reduced, where with $D=p^{2}+q^{2}$,

$$
\tilde{\eta}_{0}=((2 a+1) q-p+\sqrt{D}) / 2 q=a+\frac{p}{p-q+\sqrt{D}}=a+1-\frac{p}{p+q+\sqrt{D}},
$$

where $p \geq 2 q>0$. Hence the NSCF complete quotient $\tilde{\eta}_{1}$ is given by

$$
\tilde{\eta}_{1}=(p+q+\sqrt{D}) / p=2+\frac{2 q}{p-q+\sqrt{D}}=3-\frac{3 p-4 q}{2 p-q+\sqrt{D}} .
$$

Case 1. Assume that $k^{\prime}=1$. Then $\tilde{\eta}_{0}=\tilde{\eta}_{1}$ and $p=2 q$. Then $\tilde{\eta}_{0}=(3+\sqrt{5}) / 2$. Then because

$$
\tilde{\xi}_{u}=(3+\sqrt{5}) / 2=2+\frac{2}{1+\sqrt{5}}=3-\frac{2}{3+\sqrt{5}},
$$

and noting that $\tilde{\xi}_{u+1}>(1+\sqrt{5}) / 2$, we have $\tilde{\xi}_{u+1}=(3+\sqrt{5}) / 2$ and we have an OCF cycle of length 1 .

We can now assume that $p>2 q$. Then $2 q<p<3 p-4 q$, so for the NSCF, we take the positive representation in (8.3), with $\tilde{\eta}_{2}=(p-q+\sqrt{D}) / 2 q$.

Case 2. Assume that $k^{\prime}=2$. Then $\tilde{\eta}_{0}=\tilde{\eta}_{2}=(p-q+\sqrt{D}) / 2 q$ and $(2 a+1) q-p=$ $p-q$, or $p=(a+1) q$. Hence $p=a+1, a>1$ and $q=1$. Then $\tilde{\eta}_{0}=(p-1+\sqrt{D}) / 2$ and $\tilde{\xi}_{u}=(p-1+\sqrt{D}) / 2$. This was dealt with in Lemma 4.10 and $\tilde{\xi}_{u}$ starts an OCF period of length 4 .

Case 3. Assume that $k^{\prime}>2$. By (8.2), if $\epsilon_{u+1}=-1$, then $\tilde{a}_{u}=a+1$ and

$$
\begin{aligned}
& \tilde{\xi}_{u+1}=\tilde{\eta}_{1}=(p+q+\sqrt{D}) / p, \quad \tilde{a}_{u+1}=2, \quad \epsilon_{u+2}=1, \\
& \tilde{\xi}_{u+2}=\tilde{\eta}_{2}=(p-q+\sqrt{D}) / 2 q .
\end{aligned}
$$

However, if $\epsilon_{u+1}=1$, then $\tilde{a}_{u}=a$ and

$$
\tilde{\xi}_{u+1}=(p-q+\sqrt{D}) / p=1+\frac{p}{q+\sqrt{D}}=2-\frac{2 q}{p+q+\sqrt{D}} .
$$

The OCF partial quotient $\tilde{a}_{u+1}>1$, so $\tilde{a}_{u+1}=2, \epsilon_{u+2}=-1$ and

$$
\tilde{\xi}_{u+2}=(p+q+\sqrt{D}) / 2 q=\tilde{\eta}_{2}+1
$$


Now it cannot be true that $\tilde{Q}_{u+3}^{\prime}=\tilde{Q}_{u+3}^{\prime \prime}$, as this would imply that

$$
\tilde{\eta}_{2}=\left(p-q+\sqrt{p^{2}+q^{2}}\right) / 2 q=\left((2 A+1) Q-P+\sqrt{P^{2}+Q^{2}}\right) / 2 Q,
$$

where $P \geq 2 Q>0$. Hence $Q=q, P=p$ and $(2 A+1) Q-P=p-q$. Hence $p=A+1$, $q=1, p>2$ and $\tilde{\eta}_{2}=\left(p-1+\sqrt{p^{2}+1}\right) / 2$, which has NSCF period 2 , which gives the contradiction $k^{\prime}=2$.

Hence in both cases, the OCF and NSCF subsequently agree until we reach $\tilde{\xi}_{u+k^{\prime}}=\tilde{\eta}_{k^{\prime}}$ if $k^{\prime}$ is odd, or $\tilde{\xi}_{u+k}=\tilde{\eta}_{k}$ if $k^{\prime}$ is even, where $k=k^{\prime}$ or $k^{\prime} / 2$, according to whether there are one or two occurrences of an equation of type (8.1).

From the NSCF period properties of Lemma 7.1(d) and [1, Section 5.6.3(ii), p. 113], we have the NSCF period extract

$$
\tilde{\eta}_{0}=a+1-\frac{1 \mid}{\mid 2}+\frac{1 \mid}{\mid a}+\frac{y \mid}{\mid \ldots} .
$$

If the OCF makes the choice $\tilde{\xi}_{u+1}=\left(p-q+\sqrt{p^{2}+q^{2}}\right) / p$, then from (8.4), the OCF makes the same choice at $\tilde{\xi}_{u+2}$ as the NSCF does at $\tilde{\eta}_{2}$ and hence $\tilde{a}_{u+2}=a+1$. Then we have the OCF period segment with reversed triad:

$$
\cdots+\frac{x \mid}{\mid a}+\frac{1 \mid}{\mid 2}-\frac{1 \mid}{\mid a+1}+\frac{y \mid}{\mid \ldots} .
$$

Thus we eventually get $\tilde{\xi}_{u}=\tilde{\xi}_{u+k^{\prime}}$ and this turns out to signify either an OCF period or half-period, as we now demonstrate. We now consider the various possibilities for when we have (i) one case or (ii) two cases of $\left(p+q+\sqrt{p^{2}+q^{2}}\right) / p$, with $p>2 q>0$, occurring in a NSCF period.

From Corollary 6.2, we saw that $\tilde{Q}_{u+1}^{\prime}=\tilde{Q}_{u+1}^{\prime \prime}$ implies that

$$
H\left(\frac{u_{u}}{v_{u}}\right)>H\left(\frac{u_{u}+r_{u-1}}{v_{u}+s_{u-1}}\right) \Longleftrightarrow(-1)^{u} \epsilon_{1} \cdots \epsilon_{u} t=1,
$$

where $t=\operatorname{sign}\left(Q_{0}\right)$. So if $k^{\prime}$ is the NSCF period length, $\tilde{Q}_{u+k^{\prime}+1}^{\prime}=\tilde{Q}_{u+k^{\prime}+1}^{\prime \prime}$ implies that

$$
H\left(\frac{u_{u+k^{\prime}}}{v_{v+k^{\prime}}}\right)>H\left(\frac{u_{u+k^{\prime}}+r_{u+k^{\prime}-1}}{v_{u+k^{\prime}}+s_{u+k^{\prime}-1}}\right) \Longleftrightarrow(-1)^{u+k^{\prime}} \epsilon_{1} \cdots \epsilon_{u} \epsilon_{u+1} \cdots \epsilon_{u+k^{\prime}} t=1 .
$$

However, in case (i), from [1, Section 5.6.3, p. 113], we have $\epsilon_{u+1} \cdots \epsilon_{u+k^{\prime}}=-1$ if $k^{\prime}$ is even and the OCF period consists of a doubling of the NSCF period, with consecutive halves differing by a reversal of a triad. This is also the situation if $\epsilon_{u+1} \cdots \epsilon_{u+k^{\prime}}=1$ and $k^{\prime}$ is odd. Whereas if $\epsilon_{u+1} \cdots \epsilon_{u+k^{\prime}}=-1$ and $k^{\prime}$ is odd, we see that the OCF period is essentially the same as the NSCF period, apart from a possible triad reversal. Using the equation L-NSCF $+\mathrm{N}-\mathrm{NSCF}=\mathrm{L}-\mathrm{RCF}$ (see [6, Theorem (6.5)]), where N-NSCF is the number of instances that $\epsilon_{i}=-1$ in the NSCF period, we arrive at the formulation in Theorem 8.1(b).

In case (ii), from Lemma 7.1(e), we have $\epsilon_{u+1} \cdots \epsilon_{u+k^{\prime}}=1$, so the OCF period is derived from the NSCF period by at most two reversals of triads. 
If $\xi_{0}=\sqrt{D}$, Ayyangar proved that there are two types of NSCF expansions, where Type I has no complete quotient of the form $\left(p+q+\sqrt{p^{2}+q^{2}}\right) / q$, with $p>2 q>0$, while Type II has one complete quotient of this type in a least period (see [1, pp. 110114]). Then in view of the remark following Corollary 6.2, we have the following result of Selenius.

Corollary 8.5 [7, Satz 38]. The OCF expansion of $\sqrt{D}$ is identical to the NSCF expansion in the case of a Type I NSCF expansion. However, in the case of a Type II NSCF expansion, a period of length $2 h$ is formed by juxtaposing two copies of the NSCF period, where one copy is changed by replacing the central portion

$$
\frac{\epsilon_{h-1} \mid}{\mid a_{h-1}}-\frac{1 \mid}{\mid 2}+\frac{1 \mid}{\mid a_{h-1}-1}
$$

by

$$
\frac{\epsilon_{h-1} \mid}{\mid a_{h-1}-1}+\frac{1 \mid}{\mid 2}-\frac{1 \mid}{\mid a_{h-1}} \text {. }
$$

\section{An OCF algorithm based on the NSCF algorithm}

We now describe an algorithm for finding the OCF of a quadratic surd as far as the end of a period. This algorithm eventually bypasses the bottleneck of the original OCF algorithm of Bosma in Definition 2.1, where in step (2.3) a second integer part has to be calculated. The algorithm starts off as the Bosma algorithm, but soon switches to the NSCF, incorporating the differences noted in Theorem 8.1.

First find $k$ such that $s_{k}>\left|Q_{0}\right|, \tilde{\xi}_{k}$ is NSCF reduced and either $\epsilon_{k}=1$ and $\tilde{P}_{k}<\sqrt{D}$ or $\epsilon_{k}=-1$ and $\tilde{P}_{k}>\sqrt{D}$. This will start an OCF cycle of length $k^{\prime}$ or $2 k^{\prime}$. Record $s_{0}=(-1)^{k} \epsilon_{1} \cdots \epsilon_{k} t$, where $t=\operatorname{sign}\left(Q_{0}\right)$.

Now loop on $i \geq k$ : if $\tilde{Q}_{i+1}^{\prime} \neq \tilde{Q}_{i+1}^{\prime \prime}$, calculate $\tilde{\xi}_{i+1}$ as the NSCF successor of $\tilde{\xi}_{i}$.

Otherwise $\tilde{\xi}_{i}$ has the form $(P+\sqrt{D}) / Q=\left((2 a+1) q-p+\sqrt{p^{2}+q^{2}}\right) / 2 q$, where $p \geq 2 q>0$ and $\tilde{\xi}_{i+1}=\left(p \pm q+\sqrt{p^{2}+q^{2}}\right) / p=\left(P_{1}+\sqrt{p^{2}+q^{2}}\right) / p$.

Now compute $q, p$ and $a$ :

$$
q=Q / 2, \quad p=\sqrt{D-q^{2}}, \quad a=\frac{1}{2}(-1+(p+P) / q),
$$

where $P_{1}, \tilde{a}_{i}$ and $\epsilon_{i+1}$ are determined as follows. Let $s=(-1)^{i} \epsilon_{1} \cdots \epsilon_{i} t$. Then:

(i) if $s=-1$, choose $P_{1}=p-q, \epsilon_{i+1}=1$, and $\tilde{a}_{i}=a$;

(ii) if $s=1$, choose $P_{1}=p+q, \epsilon_{i+1}=-1$, and $\tilde{a}_{i}=a+1$.

Eventually we find $k_{1}$ with $\tilde{\xi}_{k_{1}}=\tilde{\xi}_{k}$. Suppose that $\tilde{Q}_{i+1}^{\prime}=\tilde{Q}_{i+1}^{\prime \prime}$ occurs only once. Then if $k_{1}-k$ is even, or else $k_{1}-k$ is odd and $(-1)^{k_{1}} \epsilon_{1} \cdots \epsilon_{k_{1}} t \neq s_{0}$, we keep going until we reach $k_{2}$ where $k_{2}-k_{1}=k_{1}-k$, to get an OCF period of length $k_{2}-k=$ $2\left(k_{1}-k\right)$. If these conditions are not satisfied, we have reached an OCF period of length $k_{1}-k$.

See http://www.numbertheory.org/php/ocf3.html for a BCMATH implementation. 


\section{Acknowledgements}

I am grateful to Jim White for introducing me to the papers of Selenius, Ayyangar, Bosma, and Bosma and Kraaikamp, and for pointing out the relevance of the function $H$ in deriving the OCF from the RCF. I am indebted to John Robertson for noticing that in Theorem 8.1(b) I had omitted one case, and for pointing out its connection with L-RCF.

\section{References}

[1] A. A. K. Ayyangar, 'Theory of the nearest square continued fraction', J. Mysore Univ. Sect. A 1 (1941), 97-117.

[2] W. Bosma, 'Optimal continued fractions', Indag. Math. 49 (1987), 353-379.

[3] W. Bosma and C. Kraaikamp, 'Optimal approximation by continued fractions', J. Aust. Math. Soc. Ser. A 50 (1991), 481-504.

[4] J. F. Koksma, Diophantische Approximation (Springer, Berlin, 1936).

[5] K. R. Matthews, 'On the nearest square continued fraction expansion of $\left(p+q+\sqrt{p^{2}+q^{2}}\right) / p, p>$ $2 q>0$ ', see http://www.numbertheory.org/continued_fractions.html.

[6] K. R. Matthews and J. P. Robertson, 'Period length equality for the NICF and NSCF expansions of a quadratic surd', Mat. Glasnik 46 (2011), 269-282.

[7] C.-O. Selenius, 'Konstruktion und Theorie halbregelmässiger Kettenbrüche mit idealer relativer approximation', Acta Acad. Abo. Math. Phys. 22 (1960), 3-77.

KEITH R. MATTHEWS, Department of Mathematics, University of Queensland, Brisbane 4072, Australia

and

Centre for Mathematics and its Applications,

Australian National University, Canberra, ACT 0200, Australia

e-mail: keithmatt@gmail.com 